\title{
Investigation on the Sensing Properties of Organic Field Effect Transistors Using P3HT
}

\author{
Silpa S. Prasad ${ }^{\mathrm{a}, 1}$, Divya $\mathrm{G}^{\mathrm{b}}$, Sindhu $\mathrm{S}^{\mathrm{b}}$ and Shreekrishna Kumar $\mathrm{K}^{\mathrm{b}}$ \\ ${ }^{a}$ Dept of ECE, College of Engineering, Govt. of Kerala, India \\ ${ }^{b}$ School of Technology and Applied Sciences, Mahatma Gandhi University Kerala
}

\begin{abstract}
The humidity sensing behavior and the properties of P3HT based thin film organic field effect transistor (OFET) has been reported. Layers with $(\mathrm{Au}) / \mathrm{P} 3 \mathrm{HT} / \mathrm{SiO}_{2} /(\mathrm{Au})$ are coated and OFET is fabricated. The XRD pattern of thin films are plotted which shows an orientation of (100) indicating its crystalline nature. The morphological studies report the presence of dreg like structures that can absorb water molecules from a humid environment which can be used in humidity sensing applications.
\end{abstract}

Keywords: $\mathrm{P} 3 \mathrm{HT}, \mathrm{SiO}_{2}$, OFET, spin coating, sensing

\section{Introduction}

The organic electronics [3] has gained much interest with many approved advantages such as light weight, ease of processability and less fabrication costs for its devices. Organic electronics deals with organic devices that comprises of both organic semiconductors and organic dielectrics. Among them, the organic semiconductors are basically organic materials having properties that lie in between conductors and insulators. They exhibit various properties such as absorption, conduction, etc, when subjected to annealing also. If perfect analysis is done, the form of organic polymer can be changed and hence its properties too. These characteristic natures of the organic semiconductors or dielectrics can be used for many implementations, instead of using the various inorganic materials with high cost of fabrication. The thin film technology [1][12] is a novel form of "self organizing" micro-nano advancement. It plays a significant role in all the micro - nano level structures. Any thin film organic transistor [3][11] is same as a normal field effect transistor (FET). Here, within an organic FET, we have introduced an organic polymer as the active layer. The current in the organic polymers are due to the majority carriers, and there is no inversion regime. The current flow is due to the accumulation of charge carriers [10]. In this work, a study on the application of P3HT OFETs for humidity sensing is investigated. The Organic Field Effect Transistor (OFET) which is shown as in the fig. 1. is a three terminal device having its channel as P3HT thin film [12] that exhibits highest mobility. The different layers of OFET comprises of $(\mathrm{Au}) / \mathrm{P} 3 \mathrm{HT} / \mathrm{SiO}_{2} /(\mathrm{Au})$. Here the organic materials conduct current flow using majority carriers and through the pro-

\footnotetext{
${ }^{1}$ Silpa S. Prasad, Department of ECE, College of Engineering, Govt. of Kerala, India

Email: silpasiv.p@gmail.com
} 
cess of accumulation [10]. The processing characteristics of OFETs make them better candidates in their contest for area, flexibility, speed of operation and less fabrication costs. The OFET performance can be assessed in connection with mobility and on-off current ratio. The mobility values of OFET range from $0.1-2 \mathrm{~cm}^{2} / \mathrm{Vs}$ and are commendable with the values of amorphous-Si (a-Si). The on/off current ratio for OFET ranges from $10^{3}-10^{8}$.

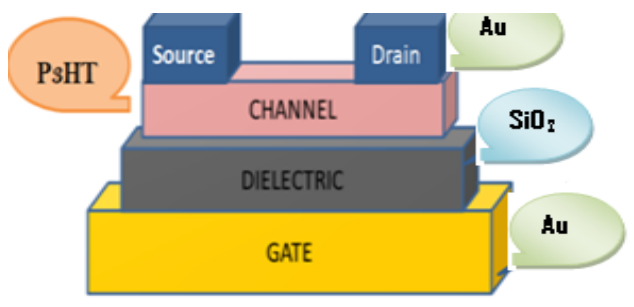

Figure 1. Basic model of an FET

Poly (3- hexylthiophenes) or P3HT is an organic polymer with excellent solubility and processability. The P3HT has a chemical structure as shown in the fig 2 . Among the various pi- conjugated polymeric materials, $\mathrm{P} 3 \mathrm{HT}$ is an interesting candidate in the organic industry. It is highly soluble in almost all organic solvents and possesses better mobility and crystallinity. The optical studies on P3HT report a band gap space within 1.7-2.1 eV. It can be dissolved in chloroform solution with a $3 \mathrm{mg} / \mathrm{ml}$ weight and is then heated to $60^{\circ} \mathrm{C}$. With the help of a magnetic stirrer, it is then stirred in ultrasonic bath to obtain a homogeneous solution. Spin coating of P3HT layer is done on the glass substrate at $500 \mathrm{rpm}$ for 15 seconds. Annealing is done on the spin coated specimen at $90{ }^{\circ} \mathrm{C}$ for about 9 minutes.

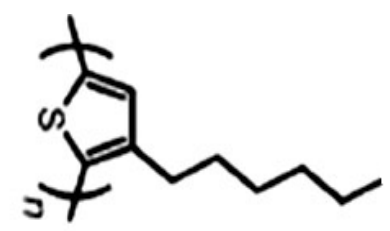

Figure 2. Structure of P3HT

\section{Spin Coating}

In this process, the glass substrate is made to spin around an axis that is straight perpendicular to the area to be coated. The spinner is designed so as to coat very thin films of liquid on the surface of wafer. The process comprises of four stages- deposition, spin up process, spin off process and evaporation time. Initially the powder to be coated is dissolved in chloroform to form a liquid solution. With the help of a nozzle, an excessive amount of the liquid solution is sprayed onto the centre of the substrate surface in order to avoid irregularities while coating. The solution is sonicated for a required amount of time at a fixed temperature, before being stirred for 24 hrs. This is the spin up process, in which the spin speed reaches its final speed. A 
centrifugal force is formed resulting in a perfect coating on the substrate. Then, the film is dried to get some portion of the solvent to evaporate, which is called the spin off process. The wafer thickness is being controlled by the spinning speed, spinning time and solution viscosity. Any thickness can be coated using the spin coating method as the thickness of the film is inversely proportional to the square root of the speed of spinning. In this technique the film thickness can be altered by changing the spin speed and uniform film thickness can also be acquired.

\section{Sputtering}

Sputter deposition technique is one of the most widely used physical vapor deposition methods having many advantages over other deposition methods such as pulsed laser deposition and molecular beam epitaxy methods. This technique is very much efficient in case of the preparation of coatings on very large-area films. In this technique particles get vaporized from a surface or target, through the sputtering process. Sputtering is a vaporization method where the target atoms are expelled from a solid surface by the energy transfer from a gaseous ionic particle from the plasma. The Hind Hivac Planar magnetron Sputtering Unit used for the sputtering process. The energetic ions are bombarded on a target in vacuum using plasma with low pressure of $<5 \mathrm{~m}$ Torr. As the distance between the substrate and source is very small compared to the thermal evaporation, the particles after undergoing sputtering process, experience only few collisions between the target and substrate. The source of sputtering can be anything like a mixture, compound or alloy which is being vaporized along with the bulk of the target space. The sputtering source is arranged such that it can be vaporized in any direction. Sputtering technique is mainly used for the preparation of thin film coatings for various applications such as reflective coatings, solar panels etc.

\section{OFET Fabrication}

For the process, the source-drain electrodes are being coated using sputtering technique from a gold layer onto a Silicon ( $\mathrm{Si}$ ) substrate. The channel or semiconductor layer is spin-coated with poly (3-hexylthiophene) P3HT soluble in chloroform. and was procured from Sigma Aldrich. Finally, the gate electrode is patterned on the top using gold $(\mathrm{Au})$. The dielectric layer is patterned with Silicon dioxide (SiO2).

\section{Morphological Analysis}

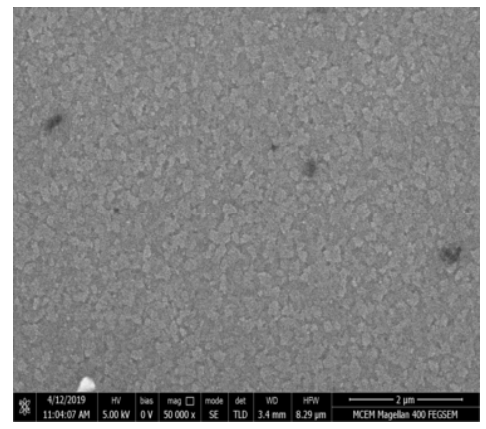

Figure 3. a. SEM of P3HT film

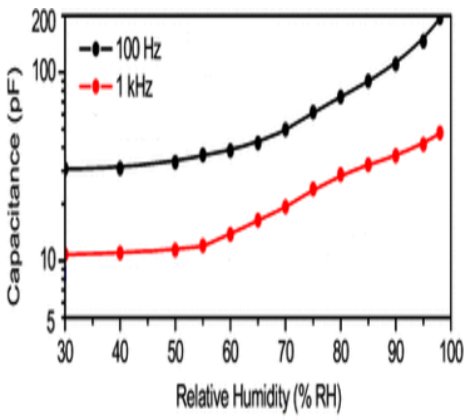

b. Response pattern for humidity sensing 
The morphological analysis [7][8] of the P3HT thin film is done using SEM analysis. A pure crystalline nature for P3HT can be attained. After the annealing process, SEM analysis shows the cluster of nanofibers $[12,13]$ in P3HT thin films. This can improve the crystalline nature [11]. As the temperature of the substrate is changing, size and shape of the fiber like structures are also changing. This steers up the growth of nano fibers [10]. This kind of growth in polymers can be utilized efficiently for a wide range of sensing properties.

\section{Sensitivity}

Here, the organic composite used here act as a dielectric sensing layer. The morphological structure obtained from the SEM analysis shows the fiber structures indicating the ability to absorbing and releasing water molecules and this in turn alters the capacitance value of the device. The sensor is opened to the presence of water vapor molecule. This results in the water molecules to get down into the porous like structures and thereby making a change in the capacitive plot of the device as shown in fig 3.b. The figure represents the connection of the OFET capacitance with the relative humidity or $(\% \mathrm{RH})$. It shows the correlation of the device sensitivity at some selected frequencies of $1 \mathrm{kHz}$, and $100 \mathrm{kHz}$ at a humidity range of about (10-90) $\% \mathrm{RH}$. The sensitivity of the device $0.072 \mathrm{pF} / \mathrm{RH} \%$ at $100 \mathrm{kHz}$ and $0.700 \mathrm{pF} / \mathrm{RH} \%$ at $1 \mathrm{kHz}$ are inversely proportional to the growing value of the shown frequencies.

\section{Conclusion}

The P3HT is an organic polymer and its thin films are prepared on glass substrates through spin coating procedure. Layers with $(\mathrm{Au}) / \mathrm{P} 3 \mathrm{HT} / \mathrm{SiO}_{2} /(\mathrm{Au})$ are prepared and an organic field effect transistor is fabricated. XRD results of P3HT confirms that film has a (100) orientation with crystalline nature. The investigation on its morphology provides a well-defined structure with fiber like nodules. The studies reports that the appearance of nano fiber like particles while exposed to the presence of water vapor, resulted in the process of the getting down of the water molecules into the porous structures and this made a change in the capacitive output of the device.

\section{References}

[1] M. Ohring, The Materials Science of Thin Films, 2nd ed. Academic Press, 2001.

[2] P. Barna .History of thin films: growth techniques characterization .

[3] W. Bruetting. Ph ysics of organic semiconductors. Wiley- VCH, Weinheim,2005.

[4] AR. Brown, C. P. Jarrett, D. M. Deleeuw, and M. Matters. Field-effect transistor made from solutionprocessed organic semiconductors. Synthetic Metals, 88:37-55, 1997.

[5] L .I. Maissel and R. Glang, Handbook of Thin Film Technology (McGraw-Hill Book Company, New York, 1983).

[6] K.L. Chopra.Thin Film Phenomena. Florida, Robert E. Krieger Publishing Company, 1979.

[7] Silpa S. Prasad, Divya K. Nair, K Shreekrishna Kumar, Microstructural \& Optical Properties of Thin Layers in the Fabrication of Field Effect Transistors. Int. J. of Science \& Research (IJSR), ISSN : 2319 - 7064. (2017).

[8] Abdullah A. Hussein, Abdullwahab A. Sultan, Mohammed T. Obeid ,Asheaq T. Ab dulnabi , Mohammed T. Ali.Synthesis and Characterization of poly(3- hexylthio phene. International Journal of Scientific Engineering and Applied Science (IJSEAS) - Volume-1, Issue-7,October 2015 ISSN: 2395-3470 
[9] U. Bielecka, P. Lutsyk, K. Janus, J. Sworakowski, W. Bartkowiak .Effect of solution aging on morphology and electrical characteristics of regioregular P3HT FETs fabricated by spin coating and spray coating", Organic Electronics 12 (2011) 1768-1776

[10] Silpa S. Prasad, K Shreekrishna Kumar .Investigation On Various Properties Of Organic And Inorganic Thin Film Transistors", Engineering Technology for Societal Development (ESD), 27th Swadeshi Science Congress, November 7-9, 2017

[11] Silpa S. Prasad, K Shreekrishna Kumar. Experimental Study on Grain Sized Crystals in the thin Layers of OFETs.International Journal of Engineering Technology Science and Research IJETSR , ISSN 2394 - 3386 Volume 4, Issue 12 December 2017.

[12] Silpa S. Prasad, K. Shreekrishna Kumar.An investigation towards the influence of grain size crystals in the thin layers of Organic FETs. 2018 2nd International Conference on Inventive Systems and Control (ICISC), 2018

[13] V. D. Ambeth Kumar,S. Malathi,Abhishek Kumar,Prakash M and Kalyana C. Veluvolu, "Active Volume Control in Smart Phones Based on User Activity and Ambient Noise" ,Sensors 2020, 20(15), 4117; https://doi.org/10.3390/s20154117 